\title{
Reliability Optimal Design of Cylindrical Torsion Spring with MIGA Algorithm
}

\author{
Hongchao $\mathrm{Wu}^{1, \mathrm{a}}$, Yueqiang Zhang ${ }^{1, \mathrm{~b}}$, Yingsheng Huang ${ }^{2, \mathrm{c}}$ and Mingsheng \\ Zhang ${ }^{2, d}$ \\ ${ }^{1}$ China North Vehicle Research Institute, Beijing 100072, China; \\ ${ }^{2}$ School of Mechanical Engineering, Dalian University of Technology, Dalian 116024, China; \\ awhoaelf@sina.com, bsyjwork@yeah.net, cethanhuangys@outlook.com, d347153192@qq.com
}

Keywords: MIGA, Cylindrical helical torsion spring, Reliability-based design, Optimal design.

\begin{abstract}
Based on the design features of cylindrical torsion spring, this paper established a multi-objective optimization mathematical model for solving the problem of nonlinear constraint, and used MIGA algorithm to optimize the design with the conditions of strength reliability. Compared to the initial design, the optimization results showed weight had reduced $62.8 \%$ and stiffness had increased $43.6 \%$, which verified MIGA algorithms and multi-objective optimization model was correct and useful in dealing with the problem of structural design and optimization of reliability.
\end{abstract}

\section{Introduction}

Luo took advantage of genetic algorithm to established valve spring optimization model and compared it with conventional optimization methods, considering strength conditions, stiffness conditions, and stability conditions as restrictions and avoiding resonance [1]. Li applied fuzzy design theory and reliability design theory to establish the mathematical model of fuzzy reliability optimal design of spring, and optimize with genetic algorithms [2]. Zhou analyzed the spring calculation and optimization mathematical model, using MATLAB software programming to complete the calculation and optimization of cylindrical compression spring to improve design efficiency and optimize the spring structure [3]. Han established the nonlinear constrained multi-objective optimization mathematical model to solve this kind of problems, and applied MSGAII algorithm to the optimization of cylindrical compression spring [4].

Currently reliability design is given little consideration in cylindrical torsion spring design, this paper established a multi-objective optimization mathematical model for solving the problem of nonlinear constraint, and used MIGA algorithm to optimize the design of cylindrical torsion spring to prove the effectiveness and practicality with the conditions of strength reliability.

\section{Reliability optimal design mathematical model of cylindrical torsion spring}

\subsection{Design Variables.}

Cylindrical torsion springs are widely used in automated machinery, there are three main independent design parameters affecting the performance of the springs, namely the total number of turns of the spring $\mathrm{n} 1$, the diameter D2, and spring wire diameter $\mathrm{d}$. Best combination between them can make the best working condition of springs, so the design variables of the cylindrical spring optimization mathematical model is $\mathrm{X}=[\mathrm{n} 1, \mathrm{D} 2, \mathrm{~d}]^{\mathrm{T}}=[\mathrm{x} 1, \mathrm{x} 2, \mathrm{x} 3]^{\mathrm{T}}$.

\subsection{Objective Function.}

The optimal objective function of cylindrical spring should maximally reflect design, manufacture and performance requirements, to maximize their economic benefits. Therefore, with the optimal combination of design parameters, we can reduce weight of the spring, increase the stiffness under the circumstance of normal operation, and finally to give full play to the performance of the 
cylindrical compression spring. Objective function of formula (1), (2) are correspond to the minimum weight and maximum stiffness respectively.

$$
\begin{aligned}
& f_{1}(X)=0.25 \pi^{2} \rho n_{1} D_{2} d^{2} \\
& f_{2}(X)=G d^{4} /\left[8 D_{2}^{3}\left(n_{1}-1.75\right)\right]
\end{aligned}
$$

\subsection{Reliable constraint of strength}

$\sigma_{\max }$ is the maximum bending stress of the cross section of spring wire. Assuming the stress and intensity of spring are normally distributed, random variable $\mathrm{T}$ is also normal distributed, so $\sigma_{\max }$ is also normally distributed with mean $\sigma_{\max }$. Standard deviation $S_{\sigma}$ and reliable coupling equation are as follows respectively [4]:

$$
\begin{aligned}
& \overline{\sigma_{\max }}=\frac{4 \frac{x_{2}}{x_{3}}-1}{4 \frac{x_{2}}{x_{3}}-4} * \frac{\bar{T}}{0.1 x_{3}^{3}} \\
& S_{\sigma}=0.08 \overline{\sigma_{\max }} \\
& \mu_{F}=\left(\overline{S_{\sigma}}-\overline{\sigma_{\max }}\right) /\left(\sigma_{S_{a}}^{2}+S_{\sigma}\right)^{1 / 2}
\end{aligned}
$$

$\overline{S_{\sigma}}$ is the material mean; $\sigma_{S_{\sigma}}$ is the standard deviation of the material

So the reliability constraint equation of the strength of the spring is:

$$
g_{1}(X)=\mu_{F} \geq \mu_{R Z}
$$

\subsection{Structural constraints}

The range of the spring design parameters is limited. Based on a cylindrical compression spring design requirements and design formulas, the design constraints can be established.

Spring index constraint:

$$
g_{2}(X)=4-x_{2} / x_{3} \leq 0
$$

Stability requirements:

$$
g_{3}(X)=\left(x_{1}-0.5\right) x_{3} / x_{2}-b \leq 0
$$

No resonance constraint:

$$
g_{4}(X)=k f_{c}-f \leq 0
$$

Mean diameter constraint:

$$
D_{2 \min } \leq D_{2} \leq D_{2 \max }
$$

Diameter constraint:

$$
d_{\text {min }} \leq d \leq d_{\text {max }}
$$

Active number of coils constraint:

$$
n_{1} \geq n_{\text {min }}
$$

\section{MIGA Algorithm}

The multi-island genetic algorithm divides the whole evolving population into several sub-groups known as the "island", and then complete selection, crossover, and variation to the sub-groups on each island independently [5]. During the process of multi-island genetic algorithm optimization, we use the initial value to optimize the operation and select some of the individuals for "transfer" operation regularly and randomly with the initial convergence achieved. Through this way, we can maintain the diversity of the population and inhibit premature. MIGA is a pseudo parallel genetic algorithm, which can optimize the field better and find the global optimal solution. 
Under conditions of design reliability of strength, this paper sets up a multi-objective optimization mathematical model to solve such nonlinear constraint problems, using MIGA algorithm to optimize the design of cylindrical torsion spring. The process is shown as Figure 1.

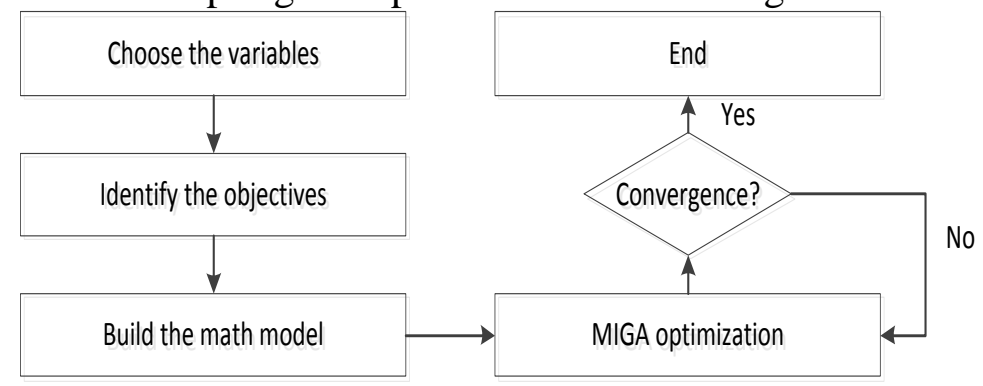

Fig. 1 Optimal design flow of cylindrical torsion spring

\section{Examples of optimization}

Taking the design of combustion engine valve springs for an example, the spring's operating frequency $\mathrm{fc}=25 \mathrm{~Hz}$, the material is $50 \mathrm{CrVA}$, the shear modulus $\mathrm{G}=81000 \mathrm{MPa}$, spring operating temperature is $200{ }^{\circ} \mathrm{C}$, the allowable stress $[\tau]=644 \mathrm{MPa}$, wire diameter $2.5 \mathrm{~mm} \leqslant \mathrm{~d} \leqslant 20 \mathrm{~mm}$, mean diameter $20 \mathrm{~mm} \leqslant \mathrm{D} \leqslant 40 \mathrm{~mm}$, active number of coils $\mathrm{n} 1 \geqslant 4.75$, operating torque $\mathrm{T}_{\max }=6 \mathrm{~N} \bullet \mathrm{m}$, $\mathrm{T}_{\min }=2 \mathrm{~N} \cdot \mathrm{m}$, the reliability requirement $\mathrm{R} \geqslant 99.999 \%$.

The comparative results of Cylindrical springs optimization are shown in Table1 (the results in the table are not yet matched with the standard value), where the original design (Scheme 1)compromised according to weight and stiffness requirements, the design met the requirements but did not achieve optimal results. The single objective optimization (scheme 2) with the minimum weight, corresponds to the minimum spring stiffness; and the optimization (scheme 3) with the minimum spring stiffness, corresponds to the maximum weight of spring, which is often used to improve the safety factor.

Table 1 Optimal results of cylindrical torsion spring

\begin{tabular}{cccccc}
\hline $\begin{array}{c}\text { Solution } \\
\text { Scheme }\end{array}$ & Total number of coils $\mathrm{n}_{1}$ & Design variables & \multicolumn{3}{c}{ Target viriables } \\
\hline 1 & 8 & 24 & 6 & 1.3478 & 151.88 \\
2 & 4.7508 & 20.0 & 3.6109 & 0.2416 & 71.7 \\
3 & 4.8610 & 34.0 & 8.4978 & 2.3281 & 431.38 \\
4 & 4.8465 & 21.1 & 5.0096 & 0.5013 & 218.08 \\
\hline
\end{tabular}

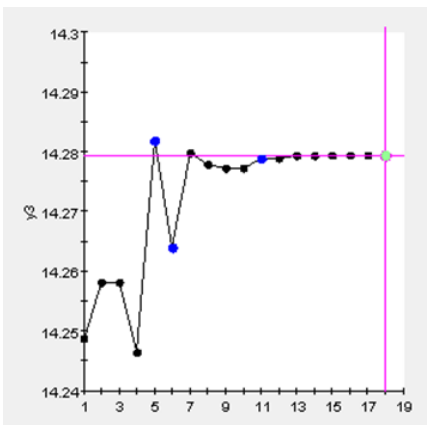

Fig.2 Reliability of strength of spring

In the process of multi-objective optimization (Scheme 4), the intensity of the spring reliability requirement $R$ is larger than 0.99999 . According to the normal distribution table $(y 3 \geqslant 4.5)$ and the changing process of $y 3$ during the optimization is shown in figure 2. Because of y3 $>14$, so it meets the reliability requirements of the design. 
In multi-objective optimization, the trend of weight and stiffness are shown in Figure 3 and Figure 4, where the weight reduced $62.8 \%$ and rigidity increased $43.6 \%$ comparing to the original design. To meet the design requirements and take the relationship between the spring weight, stiffness and strength between reliability into account, we try to look for a reasonable balance between the various sub-goals to achieve optimal design.

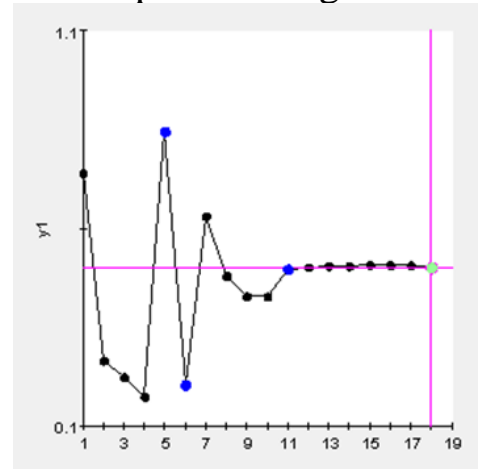

Fig.3 Weight of spring

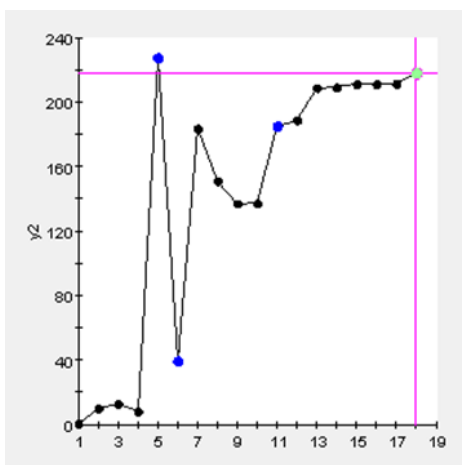

Fig.4 Stiffness of spring

\section{Summary}

This paper established a multi-objective optimization mathematical model for solving the problem of nonlinear constraint, and used MIGA algorithm to optimize the design. The MIGA algorithm is applied to the design of cylindrical torsion spring to overcome the weakness that conventional optimal design is sensitive to initial value selection and easily trapped in limited optimization. At the same time, Multi-objective optimization considers the relationship between the weight of the spring and the reliability of rigidity and strength and realizes the goal of global optimal design.

Compared to the initial design, the optimization results showed weight had reduced $62.8 \%$ and stiffness had increased 43.6\%, which verified MIGA algorithms and multi-objective optimization model was correct and useful in dealing with the problem of structural design and optimization of reliability.

\section{Acknowledgement}

This work is supported by National Natural Science Foundation of China (No. 61304206), and the Program of Liaoning Science and Research (No. L2013460).

\section{References}

[1]. Zhou Yao, Zhang Fenghua, Tian Feng, Yang Lin. Calculation and Optimization Design of Cylinder Helix Spring Based on MATLAB. Mechanical Research \& Application. Vol. 28 (2015) No. 135, p. 124-125.

[2]. Luo Min, Rui Yannia. Optimize Design of Gas Engine Valve Spring Based on Genetic Algorithm. JOURNAL OF SOOCHOW UNIVERSITY (ENGINEERING SCIENCE EDITION). Vol. 31 (2011) No. 2, p. 56-60.

[3]. Han Zeguang, FEI Ye, Zheng Xijian, Cylinder Helical Spring Conceptual Design Based on Multi -Objective Evolutionary Algorithms, Machine Design and Manufacturing Engineering, Vol. 35 (2006) No. 3, p. 27-30.

[4]. LI Xiulian, HAN Zhixin. Fuzzy reliability optimal design of cylindrical spiral compression

[5]. spring based on genetic algorithm. Journal of Machine Design. Vol. 21(2004) No. 1, p. 34-36 
[6]. Mu Shuzhi et al. Topology Optimization of a Continuum Structure Using Multi-Island Genetic Algorithm. Mechanical Science and Technology for Aerospace Engineering. Vol. 28(2009) No. 10, p. $1316-1320$ 